\title{
Sonic stimulation at certain frequencies can confer limited protection on nematode host infected with Serratia marcescens
}

\author{
Gemini Gajera, Anjali Kalla, Himani Zaveri, Pinal Sanandiya, Pooja Patel, Vijay Kothari* \\ Institute of Science, Nirma University, Ahmedabad- 382481, India \\ *vijay.kothari@nirmauni.ac.in; vijay23112004@yahoo.co.in
}

\begin{abstract}
Different mono-frequency and poly-frequency sounds were investigated for their possible therapeutic effect on infected worm (Caenorhabditis elegans) population. Sound corresponding to the frequency of $700 \mathrm{~Hz}$ and $2,000 \mathrm{~Hz}$ were found to confer $11-27 \%$ survival benefit on worm population challenged with multi-drug resistant gram-negative bacterial pathogen Serratia marcescens.
\end{abstract}

Key Words: Sonic stimulation, Caenorhabditis elegans, Serratia marcescens, bacterial infection

\section{Background}

Antimicrobial resistance (AMR) is being globally recognized by the policy makers as an immediate challenge requiring aggressive and coordinated response at the international level (Bhatia et al., 2019). In face of this serious health challenge impacting the global economy and development, novel innovative approaches not limited to discovery of new antibiotics are urgently warranted. Among the possible novel approaches, one non-invasive strategy can be testing the therapeutic potential of electromagnetic radiation or sound waves (sonotherapy) against antibiotic-resistant pathogenic bacteria. In this study, we investigated effect of a variety of mono-frequency and poly-frequency sounds on the model nematode host Caenorhabditis elegans infected with pathogenic bacteria.

\section{Methods}

\subsection{Organisms}

Caenorhabditis elegans (N2 Bristol) was used as the model host for pathogenic bacteria. This worm was maintained on Nematode Growing Media (NGM) with Escherichia coli OP50 as the feed, as previously described by us [Joshi et al., 2019]. E. coli OP50 was procured from LabTIE B.V., JR Rosmalen, the Netherlands.

Pathogenic bacteria Chromobacterium violaceum (MTCC 2656), Serratia marcescens (MTCC 97), Staphylococcus aureus (MTCC 737), and Streptococcus pyogenes (MTCC 1924) were procured from Microbial Type Culture Collection (MTCC), Chandigarh. Pseudomonas aeruginosa was taken from our internal lab collection. Of the pathogenic strains mentioned above, all the gram-negative bacteria were multi-drug resistant, and the $C$. violaceum and $S$. marcescens strains used were beta-lactamase producers. Their antibiogram, along with culture conditions and growth media has previously been reported by us [Patel et al., 2019a]. 


\subsection{Sonic frequencies}

Following sounds were used in this study:

\subsubsection{Poly-frequency sound:}

Spotted Babbler bird's voice: 2260-2712 Hz

Koel bird's voice: $602-1592 \mathrm{~Hz}$

\subsubsection{Mono-frequency sound:}

$300 \mathrm{~Hz}, 700 \mathrm{~Hz}, 1000 \mathrm{~Hz}, 2000 \mathrm{~Hz}$

Bird voices recorded at Dang Forest, Ahwa, Gujarat were obtained from Dr. Arun Dholakia, (Zoology Department, PTS Science College, Surat), and their frequency analysis was executed by performing a temporal frequency analysis (TFFT) using NCH WavePad v 7.13.

Sound beeps of each required frequency was generated using $\mathrm{NCH}^{\circledR}$ tone generator. Each of the sound files played during the experiment was prepared using WavePad Sound Editor Masters Edition v 7.13in such a way that there is a time gap of one second between two consecutive beeps of mono-frequency sounds (each beep was of 1 s duration). No time gap was there between two consecutive playing of bird voice.

\subsection{Sonic stimulation of the infected worms}

Establishment of bacterial infection in the host worm, and sonic stimulation of the infected worms was executed as described in our previous study [Patel et al., 2019b]. Spotted babbler's sound was played at the intensity of 64-78 dB, and that of Koel at 66-80 dB. Background noise was recorded to be $64 \mathrm{~dB}$. Mono-frequency sounds were played at 85.5 dB. Effect of sound treatment on infected worms was quantified through a live-dead assay, wherein number of live vs. dead worms was counted on daily basis till five days by putting the plate (with lid) under light microscope (4X).

\section{Results}

When the worms infected with different pathogenic bacteria were subjected to any of the test sounds, no statistically significant difference in the number of surviving worms was observed between the control and experimental wells (data not shown), except the cases where $S$. marcescens-infected worms were subjected to mono-frequency sound pertaining to $700 \mathrm{~Hz}$ (Figure-1A) or $2,000 \mathrm{~Hz}$ (Figure-1B). Sonic stimulation of the infected worms with $700 \mathrm{~Hz}$ sound seemed to be more effective than 2,000 Hz. Former conferred $27 \%$ ( $p<0.05)$ survival benefit on the worm population facing $S$. marcescens challenge, as compared to $11 \%$ ( $\mathrm{p}<0.001)$ offered by latter, as revealed from worm count recorded on fifth day. Protective effect offered by $2000 \mathrm{~Hz}$ sound was marginally higher on fourth day than that on fifth day. 


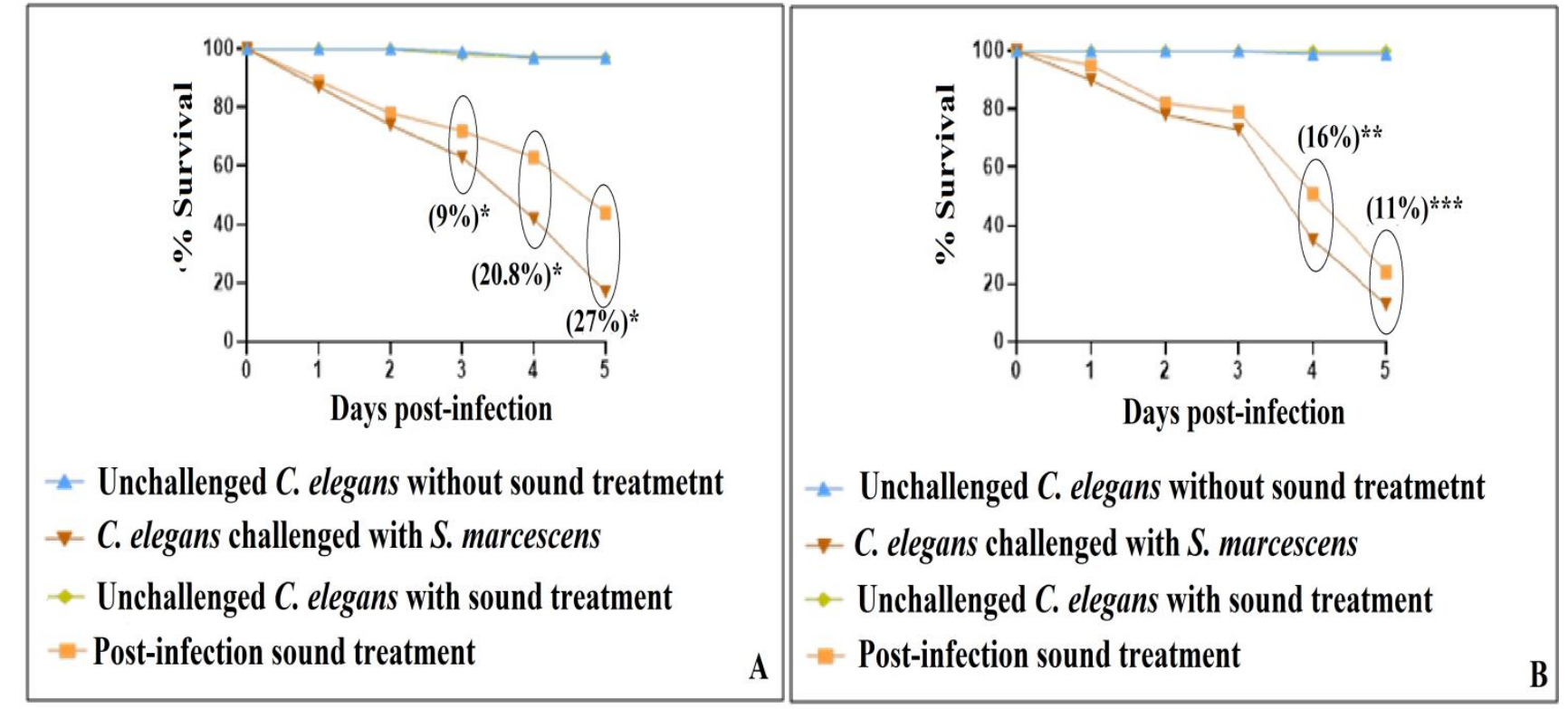

Figure 1: Protective effect of post-infection sound treatment on C. elegans challenged with $S$. marcescens

(A) (700 Hz; $85.5 \mathrm{~dB})$; (B) (2000 Hz; $85.5 \mathrm{~dB})$

Sound treatment had no effect on survival of non-infected worms; Percent values in parenthesis indicate the difference between number of surviving worm in control and experimental wells; $* \mathrm{p}<0.05, * * \mathrm{p}<0.01, * * * \mathrm{p}<0.001$;

Sound files containing $2000 \mathrm{~Hz}$ and $700 \mathrm{~Hz}$ beep are provided as supplementary material.

\section{Conclusion}

Taken together with our previous results [Patel et al., 2019b], this study indicates that certain sonic frequencies (e.g. $400 \mathrm{~Hz}, 700 \mathrm{~Hz}, 2,000 \mathrm{~Hz}$ ) can exert some therapeutic effect on worms challenged with pathogenic bacteria. This effect seem to be a function of the 'type' (e.g. frequency) of sound used, and also the pathogenic bacterial species being targeted. From the limited insight provided by our hitherto studies [Kothari et al., 2016; Kothari et al., 2018; Patel et al., 2019b] S. marcescens appear to be a sound-responsive bacterium. Challenge for future studies lies in elucidating the molecular mechanisms associated with this soundresponsiveness of bacteria, and identifying the most effective sonic stimulation (in terms of frequency and sound intensity) offering maximum protection against bacterial infections. Whether similar protective effect of sonic stimulation can be demonstrated in more complex model organisms, yet remains to be investigated.

\section{Acknowledgement}

Authors thank Nirma Education and Research Foundation (NERF, Ahmedabad) for infrastructural and financial support; and Dr. Arun Dholakia for providing bird voices recorded by him.

\section{Supplementary files}

Sound clips corresponding to $700 \mathrm{~Hz}$ and $2000 \mathrm{~Hz}$. 


\section{References}

Bhatia, R., Katoch, V.M. and Inoue, H., 2019. Creating political commitment for antimicrobial resistance in developing countries. Indian Journal of Medical Research, 149(2), p.83. https://dx.doi.org/10.4103/ijmr.IJMR_1980_17

Joshi, C., Patel, P. and Kothari, V., 2019. Anti-infective potential of hydroalcoholic extract of Punica granatum peel against gram-negative bacterial pathogens. F1000Research, 8. https://dx.doi.org/10.12688\%2Ff1000research.17430.2

Kothari, V., Joshi, C., Patel, P., Mehta, M., Dubey, S., Mishra, B. and Sarvaiya, N., 2016. Influence of a mono-frequency sound on bacteria can be a function of the sound-level. Indian Journal of Science and Technology, 2018, 11. http://dx.doi.org/10.17485/ijst\%2F2018\%2Fv11i4\%2F111366

Kothari, V., Patel, P., Joshi, C., Mishra, B., Dubey, S. and Mehta, M., 2016. Quorum sensing modulatory effect of sound stimulation on Serratia marcescens and Pseudomonas aeruginosa. Current Trends in Biotechnology and Pharmacology, 2016, 11, 121-128. https://doi.org/10.1101/072850

Patel, P., Joshi, C. and Kothari, V., 2019a. Antipathogenic potential of a polyherbal woundcare formulation (Herboheal) against certain wound-infective gram-negative bacteria. Advances in pharmacological sciences, 2019, p. 1-17. https://doi.org/10.1155/2019/1739868

Patel, P., Patel, H., Vekariya, D., Joshi, C., Patel, P., Muskal, S. and Kothari, V., 2019b. Sonic stimulation, and low power microwave radiation can modulate bacterial virulence towards Caenorhabditis elegans. Anti-Infective Agents, 17, p. 1-13. https://doi.org/10.2174/2211352516666181102150049 